\title{
HACIA UNA REVISIÓN CRÍTICA DEL CONCEPTO "GRAMATICALIZACIÓN"
}

\author{
Rivas Zancarrón, Manuel
}

Linversidad de Cádiz. Facultad de Fllosofia y Letras

Avda. Gómez Clla, sn. 11003-Cádiz (España)

Tfno.: (+34) $956015869 \mathrm{Fax}:$ (+34) 956015501 e-mal: mamel rivasancues

(Recibido, mayo 2001: aceprado, jumio 2001)

BIBIID [1133-682X (2000-2001) 8-9; 227-244]

\begin{abstract}
Resumen
Son muchas las ocasiones en las que el linguista se sirve de terminos para aclarar conceptos que no tienen una existencia real en la descripcion lingüistica. Creemos que el fenómeno de la "gramaticalización" se ajusta perfectamente a este tipo de idea. Este vocablo surge de un engaño visual que experimenta investigador al detenerse en una expresión que tiene forma de "algo", pero que ha perdido su significado originario. Aquella palabra, que mantiene el mismo aspecto de antes. cumple ahora otra función: sin embargo. debido a que no posee un significado lan "concreto", se piensa que éste se ha perdido sin dejar rastro $\%$ consecuentemente, se ha transformado en un mero "útil" gramatical. En este trabajo, revisarcmos algunas de las teorias más importantes sobre la "gramaticalizacion" y concluiremos que no tiene objeto hablar de ella.
\end{abstract}

Palabras clayes: Morfologia: Gramaticalización.

\begin{abstract}
In many occasions the linguists use terms to clarify concepts that do not have a real existence in the linguistic description. The phenomenon of the "grammaticalization" confirms this idea. The word appears in the description by a visual deceit that the investigator experiences when he studies an utterance that has form of "something" , but that has lost its original meaning. That word that maintains the same form as before seems to play a different role. Neverthcless, for it does not have a so "concrete" meaning, is thought that this meaning has totally disappeared and, consequently that has been transformed into a mere grammatical "tool".

This work aims at reviewing some of the most important theories on grammaticalization and will conclude that it docs not have object lo spcak about it.
\end{abstract}

Key words: Morphology: Grammaticalization

\section{Résumé}

Maintes fots, les grammairiens emploient des termes pour éclaircir des concepts qui n'existent pas véritablement dans le domaine de la description linguistique. Nous estimons que le mot "grammaticalisation" illustre parfaitement l'idée précitée. Ce vocable designe le "mirage" trompant le linguiste qui considere une expression comme ayant lair de "quelque chose" alors qu" elle a perdu sa signification originelle. Ces mots qui affichent la même forme que jadis assurent, à présent, une fonclion bien dillèrente. Or, comme ils n'enferment plus un sens si "concret" que par le passe. 
Io linguiste en arrive à croire qu'ils en sont complètement dépourvus et que ce sens est disparu sans laisser aucune trace. L'expression serail devenue, alors, un simple "outil" grammatical.

Nous entendons passer en revue quelques-unes des théories les plus importantes sur l'idée de grammaticalisation pour en conclure qu'à notre avis cela n'a pas de sens d'en parler.

Mots clés: Morphologie: Grammaticalisation.

0.

Cuando los fenómenos lingǘsticos transparentan complicación, el investigador suele esconder su incapacidad para describirlos on términos que aluden a conceptos vacios, pero que suenan bien. Una de estas "máscaras terminológicas" lo constituye el vocablo gramaticalización. Este "fantasma conceptual" suele vagar por diferentes castillos y el investigador cree verlos sin percatarse de que ha sido vaga ilusión, pero su miedo ante una explicación coherente es tan alto, que el espectro se manifiesta como justificante de algo que no existe, de manera que la explicación sobrenatural se convierte no en un hecho axiomático, sino dogmático, que será aceptada por otros, que ven igualmente fantasmas.

En el presente trabajo vamos a realizar una labor de "desenmascaramiento" o, si se quiere, de liberación de cadenas a un fantasma inexistente y le argumentaremos por qué cste concepto ha asustado tanto como para esconderlo en un ropaje que no le viene bien. Los gérmenes que motivan la creación del término son de carácter diacrónico, basados simplemente en el hecho de que la lengua está en constante evolución, de manera que. todo aquello que no tenga una explicación fácil en sincronía se echa a las espaldas de la diacronia. Pero claro, esto sólo nos describirá hechos pasados, nunca los presentes, que son, a la postre. los que nos interesan. Empecemos con los origenes.

1.

El concepto diacrónico del «debilitamiento semántico» es el que ha dado lugar a la formación del término que estudiamos, la gramaticalización' ${ }^{\prime}$. Bajo éste, se esconde la idea de "pérdida del significado propio" de una unidad y la consiguiente transformación de ésta en un elemento exclusivamente gramatical. En el ámbito de la lingüística desarrollada en España ha sido F. Rodríguez Adrados (1969:238) el lin-

1 Es un termino, que segun apunta Lehmann (1995:9), es desafortunado por las siguientes razones: "Firstly, the term grammaical has various meanings. [... signifies that which belongs to, is part of the grammar, as opposed to, e.g., what belongs to the lexicon, to stylistics or discourse. Apart from this, grammatical has come to mean something completcly unrelated to the notion of grammaticalization: $x$ is grammatical is an abbreviation of $x$ is grammatically conect and accordingly means that $x$ conforms to (as opposed to: is incompatible with, violates) the rules of grammar. What is particularly distressing about this ambiguity is the fact that while grammatcal may have either meaning in atributive use, it can only have the second meaning in predicative use; and yet the first meaning is needed in the predicative use which is made of it in the above explication of grammaticalization". En este sentido, derivaciones planteadas por Givon (1975) o Bolinger (1978) tales como grammaticization (en vez de grammaticalization), son desafortunadas -según Lehmann (ibidem: 9) "because we would not only have to call 'grammatic' what we always have called 'grammatical"'.

Pragmalmguistica, 8-9,2000-2001,227-244 
güista que más peso ha tenido en la consolidación de este término². Entiende la morfologización o gramaticalización como "la integración en una oposición de elementos morfológicos cuya función originaria es casi siempre diferente. En Rojo (1974:29), no obstante, parece advertirse una distinción entre "morfologización" y "gramaticalización" - conceptos igualados por Rodriguez Adrados-, pues al considerar el "verbo auxiliar" desde esta perspectiva, dice de él que "está gramaticalizado y, en algunos casos, morfologizado". Las diferencias entre estos dos conceptos no se resuelven tras la lectura de su obra ${ }^{3}$. Añade este autor que, diacrónicamente, la

2 Hasta donde llegan las investigaciones de Ch. Lehmann (1995), el tórmino gramaticalizacion parece que fue acunado y aplicado por A. Meillet en su articulo "L "volution des fornes grammaticales" (1912, en Seientia. 12 $(26), 6)$, aunque la idea posiblemente sea más antigua. Ast, en el Essai sw l'ongme des connaissances hmanes (1746), Eirenne Bonnot de Condillac intenta dar explicación a las terminaciones verbales mediante la aglutinación de los pronombres personales y nantiene que el tiempo verbal procede de la union de un adverbio temporal con la raiz. Más tarde, John Home Tooke, en su obra Epea pteroénta or the diversions of Purley (1786), llama la atención sobre el hecho de que las preposiciones dervan de nombres o verbos.

Posteriormente, August Wilhelm von Schlegel (Observations sur la langue et la littératwe provençales, 1818) compara la morfologia sintética latina con la analitica de las lenguas romances y afima de esta última (apud Lehmann (1995: 1)): "C"est une invention en quelque façon négative, que celle qui a produit les grammaires analytiques, el la méthode uniformément suivie à cel égard peut se réduire à un seul principe. On dépouille certains mots de leur énergie significative, on ne leur laisse qu'une valeur nominale, pour leur donner un cours plus général et les fairc entrer dans la partie élémentaire de la langue. Cés mots devienncnt une espèce de papiermonnaie destine a faciliter la circulation". A esto le siguen ejemplos sobre articulos, auxiliares y pronombres personales, que bien pueden fundamentar una leoria de la gramalicalización.

Años después, Wihelm von Humboldt (1822:54) determinará cuatro pasos en la definición de la "grammatische Bezeichnung": I "grammatische Bezeichnung durch Redensarten, Phrasen, Säze; II grammatische Bezeichnung durch feste Wortstcllungen und zwischen Sach- und Formbedeutung schwankende Wörter; III. grammatische Bezeichung dur Analoga von Formen"; IV. grammatische Bezcichnung durch wahre Formen, durch Beugung und rein grammatische Wörter". El punto de conexion de estos grados se concentra en la pérdida significativa y en el desgaste de los sonidos por el uso. La "foma gramatical" de la que habla Humboldt, como bien apunta Lehmann (1995:2), atañe solamente a la cara de la expresión del signo lingüistico, aunque los pasos àe arriba abarcan también la del contenido y representan los tipos morfológicos de la tipología lingüistica de su tiempo (I y o ll: aislamiento; III : aglutinacion: IV: flexión). Esta teoria recibio el nombre de Agglatinationstheorte y fue puesta en practica por Franz. Bopp en su Uber das Conjugationssystem der Sanskritsprache in Vergleichung mit jenem der griechischen, Lateinischen, persischen und gemanischen Sprache (1816) y en su Vergleichende Grammatik des Sanshit, Zend, Grechischen, Latemischen, Linaischen. Alsiavischen, Gothschen und Deutschen (1833).

Con vehemencia lue defendida esta teoría por August Sthleicher en décadas posteriores y, especialmente, por Georg von der Gabelentz en su Die Sprachwissenschafi (1901). En esta obra se manejara el ya comentado termino de la Abnutang, equivalente a lo que lingüistas de cste siglo llaman erosion.

En un marco asi surge la teoria de A Meillet (1912), en la que se asume la presencia de tres clases de palabras ("mots principales", "mots accesoires" y "mols grammaticales") con una gradación entre ellas.

Con J. Kurylowicz (1965, "The evolution of grammatical categories") se define la gramaticalizacion como un proceso en el que se avanza desde lo léxico a lo gramatical, o desde lo menos a lo más gramatical.

Por último, en el ámbito de la tipología lingitística más actual, la gramaticalización encuentra eco en autores como T. Hodge o T. Gion, cuyo pensamiento puede resumirse en la frase de este último (1971:413): "Today's morphology is yerstarday"s syntax".

3 Existen tres términos que su usan frecuentemente para describir fenómcnos parecidos, cuya delimitación podría diburjarse por la perspectiva desde la que se está analizando el objeto. Si partimos de la "forma" (sustancia formal), lo habitual es hablar de procesos de "morfologización" "gramaticalización", pero si es el contenido el 
gramaticalización se trataria de un proceso que va desde lo concrelo a lo abstracto ${ }^{4}$. Para ilustrar esta idea, nos ofrece como ejemplo la evolución del verbo ir -ya sea en francés, castellano o gallego-: al principio nos encontramos con un verbo de movimiento (vou escribir un libro) y acaba expresando "futuridad" (se non apuras, vas perdé lo tren), de tal manera que la "gramaticalización forma una senda que conduce progresivamente a la pérdida total del contenido semántico previo:

Gráfico 3. Rojo (1974:29)

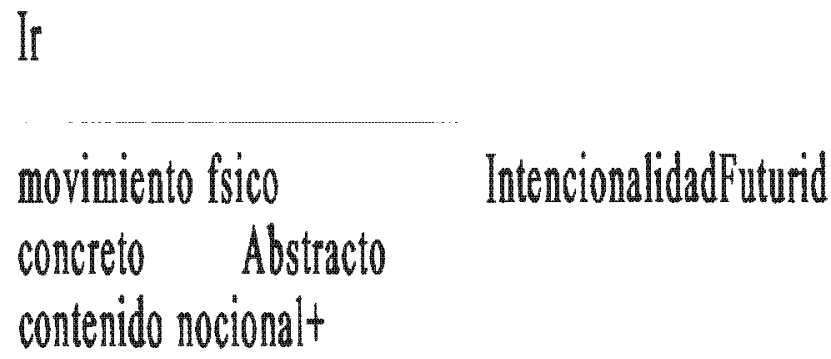

Pero, a pesar de que este esquema ${ }^{5}$ resulta válido para cualquier tipo de gramaticalización, el autor matiza que existe una diferencia entre este proceso en elementos como el francés on y el que acontece en los "auxiliares", pues en estos últimos "la existencia de un estado "posterior" no supone la anulación de los "anteriores", es decir. un valor que implica mayor abstracción convive con otro más concrelo. más cercano al punto de partida". Esta postura es aceptable desde un punto de vista sustancialista, pero creemos que no ha de

inicio del punto de mira, es entonces el término "desemantización" el que más éxito logra. El resultado en cualquier caso es el mismo -la reduccion de un elemento a lo puramente gramatical , la denominación, empero. depende del punto de partida en la aprehensión del fenómeno. En Rojo, sin embargo, parece desprenderse una matización entre "gramaticalizacion" y "morfologización" cuyos limites no son aclarados.

Por "mor lologización" entiende Lehmann (1995) o Givón (1979) la reducción de una construeción analitica a una sintética, de tal manera que los formantes gramaticales se convierten en afijos aglutinantes.

4 En la bibliografia sobre este fenomeno encontramos estudios como el de I. A. Matisoff (1991: 413) que no piensan en una relación cntre abstracción y gramaticalización, pues. segun él. "not every meaning shift $[. .$.$] is in the$ direction of greater abstraction bleaching/grammatization. Sometimes we just find 'lateral' slides in semantic space“. Además, existe de hecho abstraccion sin gramaticalizacion: es decir, aquella no es indicio de esta.

5 Este esquema de Rojo tiene algunos puntos de conexión con el que propone Guillaume (1969: 109) para explicar lo que entiende por "gramaticalización", que según él, es un movimiento iniciado en el plano de lo particular que prospera en dirección hacia lo general:

Grafico 1. Gullaume (1969:109)

\section{Stage:IIIII}

\section{Tipe of concept: SourceSource TargetTarget}


admitirse si atendemos a la función, pues aqui defenderemos que los signos lingüisticos no entienden de gradaciones, sino de "ser o no ser".

Años más tarde (1982), en su trabajo sobre «Aportaciones al estudio de la auxiliaridad», restringe Rojo este concepto, barajando una hipótesis en la que mezcla la concepción de Pottier de «incidencia directa» y nociones de gramática generativa, con el fin de distinguir entre usos auxiliares del primer elemento en un "complejo perifrástico» y usos "plenos». De esta manera, micntras que en formas del tipo
a) el alumno va a leer el libro:
b) el alumno esía leyendo el libro;
c) el alumno ha leido el libro, elc.,

no cabe más que una "conmutación léxica , en oraciones como

a) el alumno quiere leer el libro:

b) el alumno piensa leer el libro:

c) el alumno espera leer el libro, etc.

salen a la supcrficic dos frases de base (el alumno quiere algo/ el alumno piensa en algo/ el alumno espera algo), por lo que en estos últimos casos está prohibido hablar de «complejos perifrásticos», razón que le lleva, como a muchos otros lingüistas, a excluir los llamados «modales» de estos grupos. Rojo (1982:502) acota de esta mancra la concepción pottieriana del auxiliar y determina que algunos verbos que inciden sobre otros «ya sean modales (querer, poder, deber) o no, los consideraremos simplementc formas que, con mayor o menor frecuencia. llevan como complemento o predicativo una forma no personal de otro verbom. Y es aqui donde este autor (ibidem: 502) vuelve a insistirnos sobre la mayor carencia nocional del primer elemento del complejo, es decir, del auxiliar: «el verbo auxiliar necesita haber sufrido un proceso de gramaticalización que opera siempre en un sentido de pérdida progresiva de contenido semántico concreto\%. por lo que este quedará caracterizado, después de transcurrido dicho proceso. como puro instrumento gramatical.

\section{1.}

La concepción de Rojo no se aparta mucho del tratamiento tradicional de los llamados «auxiliares». Se toma como punto de partida la sustancia formal, que nuevamente vuelve a tapar los verdaderos «valores» que deberian resaltarse como forma. Hablar de «significados concrelos» o «abstractos» es tener en mente que existen palabras más significativas que otras por el hecho de que hay expresiones con una referencia real más fuerte. El significado puede ser ciertamente de divcrsa indole (categorial, léxico...), pero en todos

6 Sobre el térnino gramancalización y, discutiendo la postura de Kuryowicz. Lehmann (1995; 11) ratifica esta opción: "...one might take the position that the property of being grammatical, of belonging to the grammar, is a binary property and not a matter of degree. [..] /nyway, if this were accepted, then grammaticalization could not be a gradual, relative process. From this position it would be correct lo say that something is either grammaticalized or not grammaticalized". Esta opinión la comparten también Jakobson, Mel’cuk y Lyons.

7 Siguiendo a M. Manacorda de Roselli (1969), Rojo hace una distinción entre "conmutación lexica", en la que si sustituimos una palabra por otra no se altera ni si finción ni su articulación, aunque pueda cambiar o moditicar su significado (estoy prisionera estoy presa; estoy prisioneto estoy libre), y "conmutacion estructural", en la que se sustituye un modificador por un equivalente funcional de otra articulacion (vo estoy hbre ( en Whertah). 
los casos hemos de valorarlos concretamente: los «significados» son por naturaleza con$\operatorname{cretos}^{8}$. Argumentar, pues, grados de abstracción en el "significado" lingüistico presupone obligadamcnte una conexión con la realidad que no es deseable en el análisis de un sistema. Por otro lado, esta manera de analizar la lengua en su referencia a lo real desluce sin lugar a dudas la verdadera realidad de los hechos lingüisticos, que es otra bien distinta de la que tenemos afuera., siendo esto el primer pilar que ha de erigirse en el planteamiento de un análisis lingǘstico. La abstracción es un concepto de perspectiva y, como tal. tremendamente subjetivo, pues la decisión sobre significados más abstractos que otros obliga a una distinción entre concreto y abstracto que se manifiesta ficticia en la lengua. Considerar la palabra amor más abstracta que mesa soportaría un error sustancial -en ambos sentidos-: afirmar grados de abstracción entre palabras no significa sino una evocación de la realidad extralingüistica que carece de sentido en la descripción gramatical. Formulaciones de este tipo, que acercan la lengua a la realidad exterior, refuerzan sin ir más lejos la opinión de aquellos que creen, por cjemplo, que el sustantivo cxpresa "sustancia" o el adjelivo "cualidad".

De otro lado, como ya indicamos más arriba, excluir formas «modales» del ámbito perifrástico, supone dejarse influir de nuevo por el aspecto exterior de la palabra. Si partimos de la cxpresión (como utterance), ni la conmutación léxica ni la estructural planteadas por Manacorda de Rosetti se muestran válidas, pues hemos de insistir. una vez más, que los signos pueden resultar polisémicos o ambiguos, si analizamos tan solo la secuencia fonológica, pero, si partimos de una concepción puramente formal, olvidando todo hecho de sustancia, la anfibologia significativa queda resuelta. El caso es que en algunos de los ejemplos de Rojo ha pasado desapercibida una ambigüedad manifiesta, y ciertamente la hay, pues en absoluto podria afirmarse que sólo una posibilidad de interpretación se esconde tras una cxpresión como el alumno quiere aprobar, ya que, efectivamente, advertiremos, de un lado, un significado - para entendernos- de (verbo pleno» ( $y$ donde el conjunto se parafrasea sin lugar a dudas por «quiero algo»), pero, desde luego, lambién es posible deducir una segunda paráfrasis en la que el primer elcmento es un útil gramatical («el alumno tiene la intención de hacer algo'»). Por esta razón, ve ROJO una interpretación pasiva para cl primero de los sentidos (aprobar es querido por el alumno), pero ninguna posibilidad de transformación para el segundo. De la misma forma, no es lo mismo debe

8 Entre las premisas que incluye Trujillo (1988: 10-11) al preludio de su obra, este autor alude precisamente a la circunstancia de que los "significados" son entes concrelos: "El significado de un signo es un "objeto unico" y, por dlo, aunquc parezca paradójico, concreto. No es como creen muchos, la abstracción o la sintesis de alguna clase de objetos fisicos o mentales, sino un objeto tan real y concrelo como "este árbol" o "aquella casa", y no una pálida imagen de las cosas, como "el árbol" "la casa". "Y añade: "No puede hablarse, por ello, de diversos grados de abstracción. El "sistema" lingüistico, o saber implicito de la lengua, no es un plano abstracto, sino tan concreto como el de las cosas que vemos y tocamos".

9 La misma valoración puedc hacerse de cstudios muy recientes sobre "gramaticalizacion" cono el que presenta Heine (1993). Lo que más le preocupa a este autor es explicar qué tienen en común el auxiliar y el verbo principal para que luego se dé el "transfer" hacia una "abstract, grammatical entity". Insistimos en que las entidades gramaticales son tan abstractas o tan concretas como las lcxicas, la diferencia reside en el tipo de significado que cada una desarrolla. 
cantar, donde se induce una "obligación' a un deber "algo", con un sentido absolutamente distinto (debo mil pesetas = débito", "deuda'10).

\section{2.}

Y es que el concepto de «gramaticalización», como observa Schlieben-Lange (1971: 109), ha sido usado desde distintas perspectivas:

1. Histórica. Cuando se habla de que una «perífrasis» entra a formar parte de un solo componente, o cuando se entiende -al igual que en la fonologización de entidades-como entrada en una relación sistemática de oposiciones.

2. Se utiliza a veces como sinónimo para designar la deslexicalización de un verbo auxiliar". Roca Pons (1958: 12) menciona igualmente todos estos criterios al defender una postura "diacrónica" en la demarcación del "auxiliar". Para él, la gramaticalización es "el proceso seguido por un verbo hasta llegar a ser un verdadero auxiliar", y añade que "no siempre se llega a una pérdida completa de sentido concreto". Por tanto, según el "grado de vaciedad" significativa se podrian establecer varios grupos: a) verbos como puro instrumento gramatical (haber); b) verbos registrados como "auxiliares" y "plenos" al mismo tiempo (venir, ir, andar, etc.); c) verbos que tienen sentido independiente de la forma nominal a la que se agrega y que pueden usarse con dicho sentido sin perifrasis (hallarse, encontrarse, etc.), y d) verbos auxiliares que no experimentan pérdida de significación concreta (seguir y continuar). A pesar de que, como afirma este autor (ibidem: 15), existe "una consideración "sincrónica" y otra "diacrónica" del auxiliar", creemos que, si el punto de partida son los "grados de vaciedad", o la concreción en oposición a la generalización, éstos no son distintos del histórico.

10 Y el hecho es que Rojo (1974:30) parece intuilo al determinar que todos los auxiliares deben estar gramaticalizados, o ser semánticamente débiles en relación a su uso como verbos plenos. Con esta base, llega a concluir la invalidez. del criterio semántico para marear las fronteras entre verbos auxiliares y no auxiliares: "puede haber abstracción en usos independientes ( $v a$ de mal en peor), y, como ya hemos visto, también la forma auxiliada influye sobre la auxiliar en este aspecto". Por este motivo se da entrada al témino "semiauxiliar". Rojo coloca la instrumentalidad del verbo auxiliar en primera línea, pero como advierte Lausberg (1996: 79) "kann sich aber dennoch von der traditionellen Vorstellung, das Auxiliarverb müsse in der Periphrase etwas anderes bedeuten als in unabhängigen Verwendung, nicht lösen. So verwendet es nicht, da B in seinem Inventar galizischer Auxiliarverben nur solche Charakterisierungsverben autheten, die man als semantisch geschwäch ansehen kaun, d.h. deren Bedeutung in der Periphrase irgendwie abstrahiert erscheint" ("no puede resolverse desdc la consideración tradicional de que ol verbo auxiliar debería significar algo distinto a como lo hace en el uso independiente. Asi, no se esgrime que, en su inventario de verbos auxiliaros gallegos, solamente aparezcan aquellos verbos de caracterización que pueden verse como debilitados, i.e. cuyo significado surge abstraido de alguna forma en la perifrasis")

11 La autora enumera tres grados de lexicalización en cl scno de las perifrasis románicas, o mejor dicho, existen tres formas que fundamentan la distinción entre el uso de un verbo "pleno" y "auxiliar": 1) $A$ contiene los mismos rasgos de contenido en su uso como $A$ como en su uso como verbo pleno (comenzar, contmar,...), por lo que se aceptan dos interpretaciones $(A+$ Objeto $[-$ verbo] y $A+V I=\operatorname{sintagma}]) ; 2) A$ en su uso auxiliar pierde todos los semas hasta llegar a uno con solamente valor gramatical (la igualdad entre el lexemat y el auxiliar $A$ se mantiene en sus rasgos de contenido, que, unas veces constituye con otros el significado del verbo pleno, y otras tiene función diferenciadora en el marco de una categoria gramatical: estar, $i r, a n d a r)$, y 3 ) el auxiliar solo tiene en común con un verbo la forma, es únicamente palabra morfemática (he escrito, etc.). 
Hablar de "grados" en sincronia es poco menos que contradictorio, a no ser que tratemos con una sincronia "desincronizada" o mal entendida..

3. Se usa como designación de la escala de «disponibilidad» de una perifrasis: con cuantos más verbos se combine el auxiliar. mayor grado de gramaticalización encontraremos ${ }^{12}$.

La gramaticalización se divisa siempre como el concepto que justifica la unión léxica de un compuesto: será, pues. el argumento decisivo en la mayor parte de los estudios para la constitución de las perifrasis verbales. La mayoria de gramáticos y lingüistas creen ver una «pérdida de significación» en una determinada entidad. lo que conlleva hablar de «grados» en el verbo ${ }^{13}$. Se trata de fijar si estamos ante un verbo o un "semiverbo". o si contemplamos un "auxiliar" o un "semiauxiliar". que para el caso es lo mismo". En el mismo sentido, aunque con distinto nombre. se expresa D. Bolinger (1980:297), intentando defender por qué utiliza el término quasi-auxiliaries en vez de semi-auxiliaries: "It is

12 Esta postura es la que se refleja en Tesnière ("Théorie structurale de temps composés"). Este autor señala que una caracteristica universal de las lenguas que posen tiempos compnestos con auxiliares es la de que estos tiempos forman un sistema cohercnte paralelo a las formas simples. De aqui que formule su regla - cn otro lugar ya examinada- sobre la generación de las formas compuestas a través de las simples, en la que el rasgo gramatical es transferido al auxiliar, mientras que el verbo auxiliado soporta el significado lexico.

13 De aqui que las definiciones pretendidamente "modernas" sobre el auxiliar, dada la consideracion de grados diferentes, sean fundamenlalmente diacrónicas. Asi, Heine (1993:70) define este clemento como un "item covering some range of uses along the Verb-to-TAM chain", una definición, que según él nismo expresa. toma en consideración "the dynamics of linguistics". Esta dinámica lingüistica, desde el momento en que se determina por los usos de una cadena, cncierra una concepción diacrónica que dista mucho de ser original pues no hay gramático de los encasillados como tradicionales que no hayan determinado el auxiliar por idéntico procedimiento conceptual. aunque terminologicamente difieran en algo. Y la misma valoración puede hacerse para ambas concepciones. que son la misma los "usos" lingüsticos no determinan valores de lengua sino variaciones del signo. Por esta ralon. a pesar de que -como dice Heine (ibidem)-.. un "range of uses" may refer ideally to one single use". no ha sido capaz, sin cmbargo, "Io identify an auxiliary that has one use only" (aunque en espanol tenemos, por ejemplo. el caso de haber). Evidentemente que, viendo las cosas desde las variaciones contextuales, es dificil que logre formular un concepto unitario, pues precisamente es el contexto el que expande al infinito las posibilidades designatwas de un signo, pero sería absurdo que tomáramos a la lingüistica como una cicncia donde todo es posible dependiendo de la situación de sus elementos. Con este proceder jamás definiremos una unidad. pues el significado siempre será en ella un intojo de la posición.

En todas estas definiciones del "auxiliar" se ha procedido induct ivanente: se observan contextos y se establece la hipótesis. Y el método inductivo no es -como ya deciamos- el más apropiado para la descripcion de fenónenes de lengua, Por todo esto, cuando Heine (brdem: 3-4) en sus primeras páginas definde los presupuestos que luego pondrá colofón a sus teorias ("1. Rather than viewing language as a state, it is conceived of as a dynamic entity: and linguistic behavior is described as a process rather than a state or a product, or a historical tradition. 2 . Instead of analyzing it as a closed, self-contained system, language is interpreted as an entity that is constanty shaped by external actors such as cognitive forces, pragmatic manipulation, history, etc:3. The means that language cannot be explained satisfactorily with reference to linguistic variables only; rather what is required in addition are extralinguistic parameters relating to how we perceive the world around us, an how we utilize the linguistic resources avalable to us to conceptualize our experiences, and to communicate successfully") y advierte que estos principios no son nuevos, sino que se encontraban ya formulados por lingüistas decimonónicos como Wihelm von Humboldt o Georg von der Gabelentz, se cquivoca. Ni el primero podria cstar de acuerdo con una explicación lingüistica por "variaciones" - pues qué scntido tendria entonces su innere Sprachform., ni el segundo admitiria descripciones por axiomas sustancialistas - ya que en su obra comenta repetidamente que no llevan a ningun lado-, y no en vano estos autores se consideran. por estas razones. precedentes del cstructuralismo linguistico. 
equally an error to try to create a category of "semi-auxiliaries", implying that such a category would be amcnable to its own special rules. This is why I have chosen to speak of quasi-auxiliaries". Y es que ciertamente la unión terminológica "verbo auxiliar" está mal formulada, y podria resultar tan desconcertantc como compucstos de designación categorial tales que "nombre adjetivo" o "nombre sustantivo". Es esa desviación del pensamiento hacia el mundo de la lógica la que engendra estos abscesos catcgoriales; la manera de extirparlos o hacerlos desaparecer no es otra que la de orientar la mirada hacia dentro y no hacia el exterior. Hablar de "verbo auxiliar" implica naturalmente no tener claro si cstamos ante un "verbo", ante un "auxiliar" o ante una especie distinta, y todo por ver en una "forma" algo que, desde el punto de vista del contenido, no conecta con la idea fundamental de la categoría dominante: el auxiliar tiene la apariencia del verbo, pero no expresa el contenido que se espera de él. Igual ocurre con la unión "nombre sustantivo" o "nombre adjetivo", formulada al observar que una palabra como, por ejemplo, médico puede incluirse en el ámbito de dos categorias distintas. Extraer conclusiones como que el sustantivo se refiere a sustancias o el adjetivo a cualidades, que parten de una consideración lógico-real y que luego obligan a identificar ese contenido exterior con una forma determinada, y a verse las más de las vcces condenadas a la contradicción por el brote de múltiples contraejemplos (sustantivos que expresan "cualidades' ['belleza", ,..], sustantivos que designan "accion' ["destrucción",...]. es tan absurdo como inducir a través de la sustancia formal entidades que tienen forma de verbo y agruparlas en una categoria que sólo comparte de tal la apariencia. De ahí las especificaciones terminológicas que se agregan a una categoria concreta (verbo auxiliar, nombre adjetivo, elc.).

1.3.

En esta misma línca de comprensión del (auxilian» como un útil gramatical se muestra Guillaume, ya que lo ve como un verbo especial con un significado que lo relega a la función de instrumento. La subducción en este autor se configura como un proceso equiva-

Los principios de Heine - y de todos los que como él piensan-tienen su justificación en un análisis de la lengua que ni aporta nada nuevo - pues es lo quc la tradición ha venido y sigue diciendo hasta ahora ni nosotros podemos compartir -pues parcializa el objeto de estudio-. Heine no puede conectar, como él pretende, con las formulaciones de Humboldt o Gabelentz pues estas son contradictorias con las leyes del positivismo lingüistico, y aquel autor es tanto o más positivista que la Sprachgeschchte de Hermann Paul. No debe interpretarse en un nivel histórico el concepto de "enérgeia" humboldtiano y mezclarlo con el de "forma interior del lenguaje", pues el primero es dinámico tanto histórica como universalmente, y cl scgundo sólo afccta al producto en un sistema concreto: una cosa es decir que la lengua o las lenguas sean "creativas" con respecto ana "dinamis", y otra cosa bien distinta el que las lenguas deban describirse partiendo de la "enérgeia", puesto que dichos conceptos se encuentran en planos de descripción diferentes.

14 Hablar de "semiauxiliares" comporta una confusión entre sustancia y funcion, que conduce muchas veces a hablar de poliscmias y ambiguedades. Asi, hay expresiones que pueden funcionar o como "verbo pleno" o como ayudante de éste. El término fue creado por la tradición gramatical, aunque úllimamente tratan de apropiárselo linguistas norteamericanos: R. W. Langacker (1978:858) diferencia entre "grammaticized" y "ungrammaticized auxilary elements"; D. Winford (1990) y P. Christie (1991) hablan de auxiliares primarios y secundarios (aunque se trata de una distinción formalista entre entes analíticos y sintéticos); L. J. Brinton (1988) refiere a una dicotomía entre "auxiliaries proper" y "quasiauxiliaries", y otros muchos como W. F. Twadell (1963), J. N. Green (1982), 1. D. Huddleston (1976), etcétera. 
lente a la «deslexicalización», aunque con una idea más fija de «traspaso de sentidos», de proceso de cmigración de un «significado» ordinario a otro trasladado, en el que se despierta su «vocation à l'auxiliarité». En definitiva, la generalidad y la concreción vuelve de nuevo como demarcador de estas unidades.

El fenómeno de la subducción tiene carácter universal. En las lenguas indoeuropeas la palabra es generada mediante dos operaciones: a) la «idéogenèse», a través de la cual la materia particular de la palabra es libcrada (la noción), y b) la «compensation», en la que se desarrolla una forma integra en lugar de la materia inicial por un defecto acontecido en esta. Asi, existe un haber de posesión que se traslada a un haber de estado resultante de un proceso pasado: esta carencia material necesila de una «compensación», por lo que la palabra instrumento obtenida por "subducción» no puede separarse de la palabra que le aporta su materia. Esta es la razón por la que haber genera una compensación material que toma cuerpo en la forma participial.

\subsection{1.}

Habriamos de preguntamos si la «subducción» se trata de un fenómeno aplicable sólo al campo de la «auxiliaridad) o si, explicado tal como lo hace Guillaume, podria formar parte de otros hochos lingüísticos paralelos. ¿Acaso no tendría «vocación de sustantivo» un adjetivo como bello en lo bello? ¿Se podría hablar en este caso de «subducción», donde la «complementation» fuera lo? Parece indudable que los "significados trasladados" de los que habla Guillaume no son desde luego los categoriales, aunque bien cabria la posibilidad de un ajuste de su teoria a éstos. De cualquier forma, esta hipótesis, además de estar formulada con caracteres muy subjetivos, no presenta un gran valor explicativo, ya que no diferencia la mayor parte de los casos de auxiliaridad. En una expresión como él viene andando desde casa, ¿cuál es la "complementación» de venir?, ¿es la forma de gerundio? Si esto fuera así. sacariamos como conclusión que un verbo como venir tiene, de un lado, kvocación de auxiliar) (cuya complementación es el gerundio), y de otro lado, funciona como verbo pleno (no es subducido, pero curiosamente presenta el mismo tipo de complementación). El criterio de la «complementación» puede convertirse en verdaderamente peligroso, pucs reduciría drásticamente el número de auxiliares. Desde luego, el auténtico «auxilian en español sería haber, pues ha sido subducido y necesita de un complemento participial, pero ¿qué status tendria venir, ir, andar, poder, deber, etc.? Obsérvese que muchos de estos verbos pueden desarrollar complementaciones idénticas tanto en el sentido «subducido» como en normal (es el caso de la frase anterior: él viene andando desde casals $)$. Esto último indica naturalmente que la "complementación" no es sólo un fenómeno que aflora cn el "auxiliar", sino que es característica de todos los verbos, otra cosa distinta es la forma con la que ésta se manifieste, y desde luego, si se considera al infinitivo, gerundio y participio como maneras de complementación de un significado subducido, habriamos de incluir entre los "auxiliares" a oir u ordenar (ordenó romper fllas; oye tocar campanas); la pega aquí es que estos verbos no poseen significados trasladados, sólo disponen de su forma. Quiero decir - e invierto aquí las

15 Naturamente que la ambigüedad se encuentra en la expresión, pero los ejemplos de los que Guillaume se sirve se situan también en este nivel.

Pragnalmgüistica, 8-9,2000-2001,227-244 
tornas-que no es característico de la "subducción" la existencia de "complementación", ¿0 no se podria hablar de ésta en oigo locar las campanas y, sin cmbargo, no cncontramos significado subducido?

En otro sentido, ¿quć consideración merecen para Guillaume construcciones como poder + infinitivo? Para empezar, aquí no encontramos «traspaso de significados», $\mathrm{y}$, en segundo lugar, nos preguntamos cuál es la complementación en este caso, pues ha de advertirse que pueden admitirse dos posibles: puedo ir al cine / puede que vaya al cine. La «subducción» pretende ser un concepto mental y psiquico pero no puede ser más "materialista»; para lo único que sirve es para definir perifrasis plenamente lexicalizadas o incluidas ya dentro de un paradigma por su frecuencia de uso. No nos queda claro si en venir, ir. andar, etc. hay subducción. pues, de un lado. cuál es el «traspaso de significados» que se produce, $y$, de otro, qué forma de complementación adquieren (ir, por ejemplo. puede complementarse por un gerundio o por un infinitivo). Conceptos, pues, como los de "traspaso", "cambio", "sustitución», "debilitamiento" tienen que ver con una concepción diacrónica errónca de los hechos lingüisticos. Los signos lingüísticos funcionan en sincronía y tienen todos cogida una posición dentro del sistema. No debemos hablar en ningún caso ni dc «debilitamiento», pues no hay "gradacioncs de fuerza» entre los significados, ni de «sustitución», ya que en un estado de lengua concreto este proceso es injustificable, y ni mucho menos de «vocación hacia algo» (concepto hermano del debilitamiento y primo de la sustitución). Hemos de advertir, por tanto, que los signos lingüísticos, inás que «desvalorizarse» se «revalorizan», pues si no fuese asi, perderian su condición de tales ${ }^{16}$. Con esto no queremos decir que estos grupos se obtengan por "conmutación", pues el sentido originario de esta operación veria frustrados los principios que la regulan, y en ese pecado, hemos de reconocerlo, caimos en otros trabajos, de lo que hoy nos arrepentimos. Trujillo (1988: 42) puede servir de crítica con un ejemplo parecido: "Afirmar que, en cuchillo agudo, agudo conmuta con aflado, mientras que en sonido agudo, lo hace con grave, por ejemplo, supone, además de ignorar el alcance de la propiedad de la conmutación, el

16 En el IV Congreso Internacional de Filologia Mispánica celebrado en Leipzig discutiamos con De Cos Ruiz (1994: 290) sobre el llamado proceso de "desemantización", y advertiamos lo siguiente: "Fil término "descmantización" está en si vacio de significado. Una unidad no pierde la capacidad significativa sino que, en todo caso, adquiere nuevos valores, una nueva posición funcional determinada por los elementos del contexto. La libertad del hablante, motivada por el deseo de "sorprender", situa un elemento lingüistico en un contomo que no le corresponde, originando efectos extranos a la lengua $y$, por consiguiente, ajenos al receptor, que tiene dificultades para descodificar el mensaje. Se ha creado un nuevo sentido, tomado no más que como un efecto de estilo pero sin valor on la lengua, aunoue puede estar presente cn ella como matiz de algo ya existente. Desde el momento en que esta variante deja de "extranar", puede decirse que se encuentra ya afineada en la lengua, posee un valor, que, consiguientemente, es distinto del de la unidad sobre la que fue creada. La llamada "descmantizacion" no es tal desemantización sino, al contrario, una semantización. esto es, la creación de un nuevo valor de una unidad. Tomando como ejemplo el caso de andar. manteniendo el significado habilual, con variación de un elemento del contexto (por ejemplo, introducción del rasgo 'inanimado' en el sujeto, rasgo que se manifestaba antes como incompatible) ya no es un "alguien" el que anda sino un "algo", Si la estructura se repite, puede llegar a convertirse en norma y la lorma coexiste, por tanto, con otras del sistema, llegando, incluso, a causa de su elemento diferencial. a adquirir un matiz distinto de la unidad que le sirvio de molde, a entrar cn ofros campos de significado, aquial de "funcionar" ("el reloj no anda"). Desde este momento, el nuevo matiz adquiere identidad propia, y su diferencia - la posicion de lo inanimado-. constiuye ahora el procedimiento que lo distingue de su molde primigenio. Tenemos dos unidades distintas. una, se equipara a cammar, la otra a fincronar. Lo "inanimado" ya no es motivado".

Pragmalinginistica, 8-9,2000-2001,227-244 
olvido de que lo que conmuta con agudo es cualquier adjetivo y no éstos con aquéllos. según los diversos sentidos contextuales. No es cierto que cada palabra conmute sólo con sus sinónimos aproximados, formando paradigmas o sistemas distintos de conmutación. porque las "afinidades" no provienen del sistema lingüistico -que permite a todos los adjetivos conmutar entre si-, sino de la situación contextual". Lo que si seguimos defendiendo es la idea de que el proceso de "desemantización" debc experimentar una "desintoxicación colectiva". en el sentido de ir perdiendo cada vez más adeptos.

\section{4.}

Pottier habla igualmente en sus escritos sobre «gramaticalización», aunquc cxpresado de otra manera. Él considera el auxiliar como un lexema convertido en gramema (de lexème cst devenu un grammème" [1974: 275]), con lo que en vouloir. por cjcmplo, ocurre un proceso de "grammémisation». Sin embargo. este fenómeno no supone el paso de un elemento a otro, sino que se acompaña de un cambio de propiedades combinatorias sobre cl término que se transforma". No obstante, la "dégradation sémique", conceplo que introduce el hispanista francés como elemento clave de la "grammémisation", no sólo cs padecida por el verbo sino que también la sufren el adjetivo y otras categorias gramaticales (ibidem: 288):

"Adjetivo-------------------Quant:

parler drôlement «avec dròlerie»

drôlement triste $=$ «très»

(auxiliar)------ $\mathrm{Vb}$ -

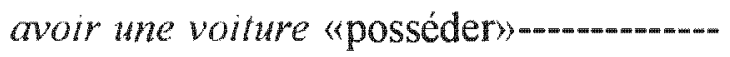

avoir fait $q q$. ch. $=$ «accompli $)$

17 Por esta razón piensa E. Gibert (1989:95) que en la concepción pottieriana del auxiliar se revela "un eritè d'ordre syntaxique". Existe desde luego una cvolución en cl concepto de "auxilaridad" on Pottier, ya que en la epoca de las Smchres grammaticales, el auxiliar quedaba definido en teminos sintacticos; en sus trabajos sobre temas espanoles son también los criterios sintáctico-distribucionales (modencla drecia) los únicos que acontecen. pero en la lingusmque generale (1974), estos criterios comienzan a maniestarse deficitarios, ya que la posibilidad que tiene una foma verbal de preceder a otra forma verbal no es suficiente para que se caracterice como atxiliar.

El proceso de grammémisation en Potier se relaciona con lo que más recientemente autores como Givon (1979) o Lehmann (1995: 13) denominan symachication, "whereby some of the lexemes assume grammatical function so that the construction may be called analytic". Una conclusion asi licne su origen en cl squema que propone Givón (1979:209) sobre las "fases de gramaticalización":

Graflo 2. Givon (1979:209)

articulierGinal

X $Y$

A.B

Pragmalngustica, 8-9,2000-2001.227-244 
1.4.1.

A lo largo de su trabajo es el criterio semántico el que triunfa en la definición del auxiliar, que es considerado como poseedor de una «charge sémique» más débil que un verbo. Pero, abandonando esta concepción, a mi modo de ver desacertada. presenta a veces pinceladas de acercamientos correctos cuando defiende que avoir, cuando es parafraseado por "posséder», se considera como verbo, pero cuando marca un estado terminado, debe ser analizado como un auxiliar. Dada esta situación, podría hablarse de un covoir y un avoir $_{2}$. Sin embargo, la semántica salpica sus ideas sobre el auxiliar, sobre todo cuando distingue entre "gramemas" y «lexemas» e indica que, por ejemplo, vouloir se encuentra a mitad de camino. Este «à mi chemin entre lexème ct gramème» se deriva no sólo de una concepción significativa sustancial del término auxiliar, sino también de una comprensión errónea de los procesos sincrónicos y diacrónicos ${ }^{18}$.

\section{5.}

La «gramaticalización» en sincronia revela la existencia de formas verbales que son portadoras tanto de significado léxico como de contenido gramatical. lo que plantea por necesidad una relación entre el léxico y la gramática. Cuando un mismo signo lingüistico -como advierte Lausberg (1996: 53)-del estilo de aller, venir. devoir, etc., puede funcionar a la vez. como morfema y lexema, entonces cabe la posibilidad de que léxico y gramática trabajen para que de los signos existentes surjan múltiples realizaciones. En cste scntido. la autora considera con acierto que la concepción tradicional de "grados de lexicalización» se muestra insuficiente, pues aquello que en linguística se acepta gencralmente como un alto grado de "deslexicalización» ("fuerte debilitamiento semántico») no es en la realidad otra cosa que un alto grado de abstracción en el significado de los signos respectivos. Así, el significado de los auxiliares consigue ser tan abstracto que puede resolverse en morfemas ${ }^{19}$. Lo que normalmente se entiende como "grado de gramaticalización" de una determinada construcción afecta a su anquilosamiento o fijeza en el sistema gramatical respectivo: pero ésta puede brotar de

18 No sólo desarrolla Potier una hpotesis semantista sobre el auxliar sino que mezcla tambien distintos niveles Este autor sufre además el peso de la tradición gramatical en la obcecada hjación sobre un termino tan poco acertado como es el de anhar. Advirtanos que, desde una perspectiva fonnalistat. Puttier excluye a deteminados auxiliares. que luego quedan incluidos cuando parte del concepto. Cuando en la Lingmstrque générale sc pintan los trazos que dibujan el auxiliar. puede determinarse una cierta confusión derivada precisamente de la mezcla de diferentes criterios. El cuadro de este fenómeno deja traslucir una foma verbal que marca una relación, una modalidad portadora de un sentido "debilitado", con "propriétés combinatoires originales" y que no rigen acusativo La pega de este mare magnmm conceptual estriba en un prestano desafortunado del término anxilion de manos de la tradicion gramatical. Asi, tambien lo schala (iibert (1989:100): "Il semble. en fait que Pottier utilise le torm "dauxiliaire" comme une étiquete préablement établie. La délinition dégagé rapelle en effet étrangement. â quelques dètails pres la définition classique de l "auxiliaire". et présente les nêmes moonenients. Cette remaque ne doit en aucun cas être comprise comme une critique de la theoris de Potticr, qui s est fixí contains objectifs el dont le propos, en tout étal de cause. nest pas de tratter de lauxiliaire mais comme une critique du concept mène "d ausilaire" qui est matheuresement souvent ulisé dans les écris de linguisles". Se llega a la conclusion de que el temino awwhat ha de ser desterrado de la descripcion lingüstica por portar precisamente un contenito sémico "debilitado".

19 Más que de "abstración". U. Lausberg deberia haber hablado de "generalizacion", pues es dificil plantear grados para el primer concepto

Pragmalingiistica, 8-9,2000-2001, 227-244 
manera independiente del grado de abstracción significativa de un signo. La linguiista señala como ejemplo que ilustra esto último el hecho de la existencia de algunos tipos de construcciones -sobre todo en los compuestos perifrásticos aspectuales- que se determinan ya como complejos unitarios anclados en un paradigma y que, además, son vistos como gramaticalizados, e independientes del grado de abstracción significativa de cada verbo aspectual en particular. Por esta razón, Lausberg (ibidem: 54) entiende por "gramaticalización" "den synchronen Vorgang, bci dem ein Zeichen grammatikalisch nutzbar gemacht wird" ("el proceso sincrónico por el que un signo se hace útil desde el punto de vista gramatical"), que se oponc a "inventarización" (Inventarisienung"2), designado como "den diachronen Etablienungsvorgang eines sprachlichen Zeichens in einem TeilSystem" "el proceso de establecimiento diacrónico de un signo lingüístico en un sistema parcial"). La autora modifica así la clasificación de los verbos auxiliares realizada por Schlieben-Lange (1971: 1): verbos que contienen en su uso auxiliar los mismos rasgos de contenido que si se usasen como verbos "plenos" (comenzar. etc), 2) verbos que pierden algunos rasgos hasta llegar a uno que lo opone en el nivel gramatical, y 3) verbos que son morfemas puros), en el sentido de que la posibilidad de un signo para ser utilizado sólo léxicamente, o léxica y gramaticalmente, o sólamente gramaticalmente queda establecida por su contenido y fijada en el sistema lingüistico. De esta manera, se prohibe concebir como perifrasis verbales construcciones de verbo-verbo cuyo significado no se obtiene por la unión de los contenidos -ya gramaticales, ya léxicos- de ambos (en oigo cantar a los niños, por ejemplo). En el discurso, los semas de los verbos que pueden usarse léxica o gramaticalmente se actualizan ya como semas léxicos, ya como semas gramaticales. Por esto.

20 La "inventarización" es verdaderamente el punto de llegada de casi todos los estudios que trabajan con el concepto de "auxiliar" y en especial de aquellos que hablan de "usos". Si la historia no ha generalizado aun una forma concreta hasta auxiliar (entendido como puro útil gramatical), en la misma noma coexistirán todas las acepciones de dicha sccuencia fonológica. Realmente iratamos aquí con manifestaciones nomativas de un mismo sistema, o, como apunta Dietrich, la sincronia del sistema implica la diacronia de la norma.

21 La idea de "Iraspaso" de semas ha sido reformulada recientemente por ciertos cognitivistas como Ileine (1993). que creen en un "transfer" de conceptos léxicos a gramaticales, donde un concepto gramatical (que puede ser d tiempo, el aspecto o la modalidad) puede ser expresado por otro léxico. El proceso es gradual hecho nada nuevo para la gramática tradicional-e implica tres estadios: "fuente" (somce), fuente y objetivo (sonre, larget), y. por último, el objetivo (target):

Grófoo 4. Heine (1993)

LevelDiscourseSyntaxMorphologyMorphophonemics

Technique Isolating $>$ Analytic < Synthetic $->$ Synthetic $-<$ Zero Agglutinating Flexional

Phase Syntactization MorphologizatronDemorphemicization Loss

\section{ProcessGrammaticalization}


más que hablar de verbos auxiliares que poseen un significado léxico fundamental que se "traspasa" 2 ", habría que hablar de realizaciones de verbos con igual valor, que tienen que ver unas veces con la "instrumentalidad" $y$, otras, con el "lexema".

\subsection{1.}

Con esta idea -que aquí compartimos-queda rechazada toda consideración del «auxiliar» como el componente enfermo de un signo. Funcionalmente, tan importante son verbos como haber en la construcción participial, que lexemas como cantar. Manifestar la existencia de «grados de valor» en un sistema es como si dijéramos de los peones en el juego de ajedrez que son elementos muy débiles. Una unidad adquiere valor desde el momento en que cumple una función dentro de un sistema concreto. Parece que, cuando se habla de «debilitamiento», se quisiera entender con ello que su papel no es decisivo, y si esto fuera asi, su presencia carecería sin duda de cualquier sentido.

\section{6.}

Pero, aun con cste panorama, el mundo de la gramalicalización descubriría un nuevo sol a finales de la década de los setenta. En honor a la verdad, más que de un descubrimiento habria que hablar de un renacer de múltiples términos en torno a cste concepto, pues este, al fin y al cabo, no se descubre, sino que se redescubre, es decir, se llega a él por los mismos procedimientos (la degradación sémica, pérdida significativa, vaciamicnto semántico, etc.), pero se le adorna con palabras que tratan de calificar al detalle cada una de sus propiedades. Estos nuevos estudios se justifican desde una perspectiva universalista - por lo que nada ha de achacárseles-, pero con pretensiones de describir sistemas históricos, que se vienen abajo por el hecho de que sus presupuestos son más ambiciosos como para detenerse en la estructura interna de una lengua concreta.

2.

Teniendo en cuenta todas estas consideraciones, puede llegarse a la conclusión de que la «gramaticalización» ha de contemplarse en un sistema de manera sincrónica, otra cosa bien distinta es que represente la "diacronía de la norma». Ésta es la postura que defendió Dietrich (1973:578) al comprender bajo este concepto el hecho de que «ein sprachliches Zeichen von der Ebene der lexikalischen Einheiten auf die Ebene der Grammatischen übergehen, d.h. instrumental und damit grammatisch distintiv werden kann. Dadurch wird entweder eine alte Opposition erhalten und nur materiell neu realisiert oder eine neue funktionelle Opposition geschaffen." ("un signo lingüístico pase del nivel de las unidades léxicas al de las gramaticales, esto es, que pueda ser distintivo desde la perspectiva instrumental y, con ello, gramatical. Por esto, o se mantiene una vieja oposición -y se realiza de nuevo matcrialmente-, o se crea una nueva oposición funcional"). Desde el punto de vista diacrónico, esto significa que un signo, en niveles lingüísticos anteriores, poseyó un significado léxico, pero, después, como realización normativa, se instrumentalizó o se gramaticalizó. 
Sincrónicamente, ha de distinguirse entre signos léxicos, que sólo en el nivel del discurso pueden designar casualmente y de forma no sistemática procesos instrumentales, y signos léxicos que cn determinadas uniones sintagmáticas expresan siempre funciones gramaticales (significado instrumental), pero que en otras uniones conservan frecuentemente significado léxico. Y esta diferenciación debe acontecer en el nivel del sistcma.

En varios trabajos de Coseriu ya quedó establecido que un objeto determinado. dependiendo del nivel estructural que se percibiese, podría ser descrito de formas diferentes: a uno y al mismo sistema (sincrónicamente) pueden corresponder diferentes normas (ya en el mismo momento del tiempo o en la sucesión de éste). Micntras algo funciona en el nivel del sistema (sincronia), pueden ocurrir diferentes manifeslaciones en cl nivel de la norma como sucesión (diacronía). Así entiende Dietrich la gramaticalización: un fenómeno que representa la «diacronía de la norma» o la «sincronía del sistema"2".

Poco sc pucde comentar más sobre este fenómeno, que, como vemos, presenta múltiples caras. La conclusión más importante que ha de extraerse es la de no pensar que los signos lingüísticos están jerarquizados por su cualidad significativa, y, sobre todo, no creer que éstos son unidades describibles desde materias visibles. La secuencia fonológica ayuda sin lugar a dudas, pero la labor del linguiista va más lcjos que la de "sistematizar" materias o hechos textuales -si es que éstos son de alguna manera sistematizables-. Son las conexiones o las posibilidades de combinaciones diferentes dentro del sistema las que nos ayudarán a cntender mojor estas construcciones. iFuera, pues, con la gramaticalización y con sus hijos y nietos ("debilitamiento semántico". "subducción", "auxiliares",...)!

22 Esta hipótesis nos la ilustra el autor con un ejemplo. Consideremos la unión habere + infm., que en una época del latin poseía el significado lćxico de "tener que hacer algo". En el discurso, sin embargo, competia con otras construcciones que se expresaban mediante morfemas $(-b o,-b s, \ldots-a m,-e s, \ldots)$. La gramaticalizacion de habere - infin. se completó en la medida en que esta construcción -en un cierto momento- comenzó a sobrevivir regularmente con un significado instrumental del mismo tipo que los que se realizaban en el sistema, $y$, con ello, pasaba a contener ol rasgo gramatical 'futuro', que era expresada en la misma noma por fomas como - bo, - bis, etc., hasta que se volvió funcional. Posteriomente, podemos encontrar junto al sistcmático habere - infin. difcrentes realizaciones nomativas, contempladas como variantes. Y mientras que las normas cambian, el sistema permanece igual, pues las oposiciones tuncionales siguen siendo las mismas. 


\section{Referencias}

BOLNGER, D. L. (1978), "Intonation across languages", en Greenberg, J. H. (ed), Universal of hman language. I. 4 vols., Stanford, pp.471-524.

BOLINGER, D. L. (1980). "Wama and the gradience of auxiliaries", en Brettschneider, G. y Lehmann, C. (eds), Wege zur Liviversalienforschung: Sprachwissenschafliche Beitrage zum 60.Geburtstag von Hansjakob Seller (Tubinger Beitrage zur Linguistik, 145), Tubinga, pp.292-299.

BRINTON, L. J. (1988), The development of english aspectual systems. Aspectualizers and post-verbal particles, Cambridge.

CHRISTIE, P. (1991), "Modality in Jamaican creole", en Edwards, W. y Winford, D. (eds), lerb phrase patterns in Black English and creoles). Dciroit, pp. 217-233.

COSERU, E. (1976). Das romanische lerbalsystem, Tubinga.

DIETRICH,W. (1973), Der periphrastische Terbalaspekt in den romanischen Sprachen, Tubinga.

GILBERT, E. (1989), "L'auxiliaire dans la théorie de Pottier". en Boucher, P. (ed), La question de l'auxiliaire, Rennes, pp.83-101.

GREEN, J. N. (1982), "The status of the Romancc auxiliaries of voice", en Vincent, N. $y$ Harris, M. Studies in the Romance verb, Londres, pp.97-138.

GIVÓN, T. (1975), "Serial verbs and syntactic change: Niger-Congo", en Li, Ch. N. (ed). Word order and word order change, Austin, Londres. pp.47-112.

GUILLAUME, G (1929), Temps et verbe. Thérie des aspects, des modes et des temps, París.

HEINE, B. (1993), Auxiliaries. Cognitive forces and grammaticalization, Nueva York, Oxford.

HUDDLESTON, R. D. (1976), "Some theoretical issues in the description of the english verb (Review of The English verb, by F. R. Palmer", en Lingua, 40, pp.331-383.

HUMBOLDT, W. von (1822), "Über das Entstehen der grammatischen Formen und ihren Einfluß aur die Ideenentwicklung", en Abhandlungen der Akademie der Wissenschaft zu Berlin (nueva edición de 1972 en Schriften zur Sprachphilosophie a cargo de A. Flimer y K. Giel), t. III, Darmstadt, pp.31-63.

LANGACKER, R. W. (1978), "The form and meaning of the english auxiliary", en Language, 54,4, pp. 853-884.

LAUSBERG, U.(1996), "Modale"lerbaadiecta?: funktionelle Untersuchung zu den franzosischen und spanischen lébalperiphrasen der Charakterisierung, Münster.

LEHMANN.C. (1995), Thoughts on grammaticalization, Múnich, Newcastle.

MANACORDA DE ROSETTI, M. (1969), "La frase verbal pasiva en el sistema español", en Estudios de gramática estructural, Buenos Aires, pp. $71-90$.

MATISSOF, J. A. (1991), "Areal and universal dimensions of grammatization in Lahu". en Approches to Grammaticalization, vol. II (ed. por E. Closs Traugott y Bern Heine en Typological Studies in Language; 19. Amsterdam/Filadelfia), pp. 383453.

MELLET, A. (1917), Linguistique historique el linguistique générale, Paris.

POTTIER, B. (1974), Linguística moderna y flologia hispánica, Madrid. 
RIVAS ZANCARRÓN, M. y DE COS RUIZ, F. J. (1994), "Construcciones con 'andar": modelo de análisis en una teoria del signo lingüistico", en lerbo e estruchuras frásicas (Actas del IV Coloquio Internacional de Lingüistica hispánica, Leipzig, 22-25 de noviembre de 1993), pp. 287-297.

ROCA PONS, J. (1958), Estudios sobre perifrasis verbales del español, Madrid.

RODRÍGUEZ ADRADOS, F. (1969), Estudios de Lingüistica general, Madrid.

ROJO, G (1974), Perifrasis verbales en el gallego achal, Universidad de Santiago de Compostela.

ROJO, G. (1982), "Aportaciones al estudio de la auxiliaridad", en Actas del II" Congreso Internacional de Hispanistas, II.

SCHLIEBEN-LANGE, B. (1971), Okzitanische und katalanische Verbprobleme, Tubinga.

TESNICRE, L. (1959), Elémenis de syntaxte structurale, Paris.

TRUJILLO, R. (1988), Introducción a la semántica española, Madrid.

TWADDELL, W. F. (1963), The English verb auxiliaries, (segunda edición), Rhode Island.

WINFORD, D. (1990), "A re-examination of auxiliary ordering Guyanese (and Jamaican). en Paper presented at the Linguistic Society of American meeting, Washington (D.C.). 